described, but in our two patients there was no other obvious cause for this condition.

At the time of their admission for myocardial infarction both patients had taken diamorphine, prochlorperazine, and glyceryl trinitrate; one patient (case 1) also took a few doses of frusemide and cyclopenthiazide, and the other required co-proxamol and buprenorphine intermittently over the first few days. None of these drugs, however, is known to cause peripheral neuropathy.

Though we may have observed no more than a chance association, we suggest that patients who complain of weakness or lethargy after a myocardial infarct should undergo careful neurological examination.

We thank Dr L D Blumhardt for arranging electrophysiological studies on these patients.

1 Hughes RAC, Winer JB. Guillain-Barré syndrome. In: Matthews WB, Glaser GH, eds. Recent advances in clinical neurology 4. Edinburgh: Churchill Livingstone, 1984:19-49.

2 Arnason BG, Asbury AK. Idiopathic polyneuritis after surgery. Arch Neurol 1968;18:500-7.

3 Morley JB, Reynolds EH. Papilloedema and the Landry Guillain-Barré syndrome. Case reports and a review. Brain 1966;89:205-22.

(Accepted 25 November 1986).

Department of Medicine, Clatterbridge Hospital, Bebington, Wirral, Merseyside L63 4JY

A J G MCDONAGH, MB, MRCP, medical registrar

J DAWSON, DM, MRCP, consultant physician

Correspondence to: Dr A J G McDonagh, c/o Dr G R Youngs's secretary; Chester Royal Infirmary, Chester CH1 2AZ.

\section{Long term exposure to hydrogen sulphide producing subacute encephalopathy in a child}

We describe a child who developed an illness resembling subacute necrotising encephalopathy after long term exposure to the toxic gas hydrogen sulphide. The appearances of long term hydrogen sulphide exposure on computed tomography have not been previously documented.

\section{Case report}

A 20 month old second child of healthy non-consanguineous parents developed intermittent paroxysmal tonic deviation of the eyes, upwards and to the right. A few months later these abnormal eye movements resolved and were followed by progressive involuntary movements of the whole body with frequent falls. His development had been previously completely normal and he had taken no drugs.

On admission he had gross truncal ataxia, choreoathetosis, and dystonia and could not stand. He was dysarthric but eye movements were normal and remained so. There was no respiratory disease.
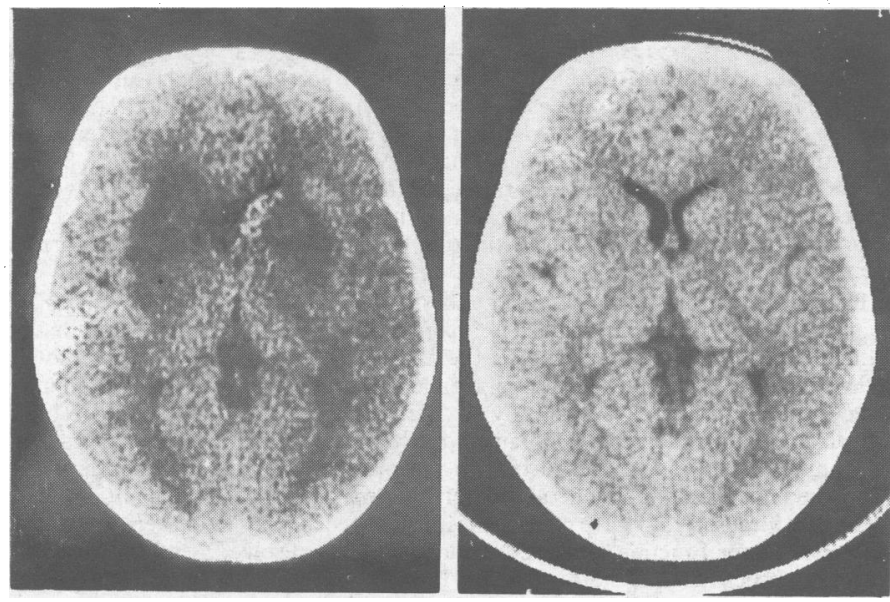

Left: initial computed tomogram shows low density areas in region of both basal ganglia and surrounding white matter. Right: a repeat scan, 10 weeks later, shows complete resolution of these areas.
Computed tomograms of the brain showed striking bilateral areas of low attenuation in the basal ganglia and in some of the surrounding white matte (figure). Negative findings on virological investigations of the cerebrospinal fluid, blood, stools, urine, and nasopharyngeal secretions and on screening for occult neuroblastoma made myoclonic encephalopathy unlikely, and there was no evidence of a recent streptococcal infection. Serum copper and lead concentrations were normal, and no abnormality was found on the neurophysiological investigations of electroencephalography, visual evoked responses, brain stem evoked responses, and peripheral nerve conduction studies. The brain scan abnormalities suggested a toxic encephalopathy. Metabolic studies included: measurements of urinary organic acids and amino acids, serum amino acids, ammonia, and lysozymal enzymes and repeated estimations of capillary blood gases and serum lactate concentrations; all gave normal results. A muscle biopsy specimen taken one month after presentation showed normal mitochondrial function with norma levels of complex 1 and cytochrome oxidase and normal mitochondrial structure on electron microscopy.

Shortly after admission the child's condition improved spontaneously, and after 10 weeks his ataxia had resolved and his choreoathetoid movements were much reduced. A repeat brain scan showed complete resolution of the abnormalities (figure)

The family lived beside a colliery, where a burning tip had been emitting hydrogen sulphide for nearly a year. Three months before the child's presentation the emissions had been monitored for four months, and the maximum recorded level in the family's housing scheme was $0.6 \mathrm{ppm}$. As the local authorities had been trying to remove the burning tip for some months before monitoring they acknowledged that levels may at times have been higher. The fire was extinguished just before the child's admission.

\section{Comment}

Several rare metabolic diseases can present with acute ataxia in childhood, and we excluded these by the relevant biochemical investigations. ${ }^{\prime}$ The most difficult endogenous metabolic disease to exclude in life is Leigh's syndrome, a subacute necrotising encephalomyelopathy which usually presents before the age of 2 years and which may show spontaneous temporary remissions. These metabolic disorders are now known to be mitochondriopathies, ${ }^{2}$ but examination of the mitochondrial respiratory chain in this child, some weeks after presentation, showed none of the recognised abnormalities.

This child had experienced long term exposure to the toxic gas hydrogen sulphide at levels of at least $0.6 \mathrm{ppm}$. Harmful concentrations and durations of exposure are known only in the context of industrial exposure in adult men, but the World Health Organisation has set community standards of $0 \cdot 003-0 \cdot 11$ ppm over 24 hours in some countries. ${ }^{3}$

Sulphide ions selectively bind to cytochrome oxidase within the mitochondria and disrupt the electron transport chain. After accidental industria exposure effects on the central nervous system are common. ${ }^{4}$ Computed tomography of the brain in a subject who died after industrial exposure showed lucent areas in the basal ganglia, as found in our patient, and these appearances suggested necrosis of the lentiform nuclei. ${ }^{5}$ Our child showed clinical improvement when his exposure to hydrogen sulphide ceased, with complete resolution of the abnormalities of the basal ganglia. All investigations for endogenous metabolic diseases gave negative results and we suggest that he developed a temporary mitochondriopathy secondary to this exposure.

We thank Dr D M Turnbull, Department of Neurology, Newcastle upon Tyne, for mitochondrial studies on the muscle biopsy.

I Chutorian AM. Neurologic emergencies in infancy and childhood. Philadelphia: Harper and Row, 1983:234-5.

2 Van Ervan PM, Ruitenbeek W, Gabreels RWO, Fischer JC, Janssen AJM. Disturbed oxidated metabolism in subacute necrotising encephalomyelopathy (Leigh's syndrome). Neuropaediatric 1986;17:28-32.

3 World Health Organisation. Environmental health criteria 19. Hydrogen sulphide. Geneva: WHO International Programme on Chemical Safety 1982:48.

4 Burnett WW, King EG, Grace M, Hall WS. Hydrogen sulphide poisoning. Review of five years experience. Can Med Assoc F 1977;117:1277-80.

5 Matsuo F, Cummings JW, Anderson RE. Neurological sequelae of massive hydrogen sulphide inhalation. Arch Neurol 1979;36:451-2.

(Accepted 8 December 1986)

Department of Surgical Neurology, Western General Hospital, Edinburgh EH4 2XU

U B GAITONDE, DMRo, clinical assistant

R J SELLAR, FRCR, consultant neuroradiologist

Department of Paediatric Neurology, Royal Hospital for Sick Children, Edinburgh EH9 1LF

A E O'HARE, MRCP, senior registrar

Correspondence and reprint requests to: Dr Sellar. 